\title{
Effect of Kangaroo Mother Care plus Touch Therapy versus Kangaroo Mother Care Alone on the Low-Birth-Weight Infant's Growth and Physiologic Responses: Randomized Controlled Trial
}

\author{
Rocamia F. Rasalan-Fermin, MD, ${ }^{1}$ Lourdes Imperial, MD² and Fay S. de Ocampo, MD² \\ ${ }^{1}$ Mariano Marcos Memorial Hospital and Medical Center \\ ${ }^{2}$ Dr. Jose Fabella Memorial Hospital
}

\begin{abstract}
Background. Kangaroo Mother Care (KMC) has facilitated infant growth and decreased morbidities and hospital stay. Adding touch therapy (TT) may potentiate the KMC benefits.

Objective. To compare the effectiveness of $\mathrm{KMC}+\mathrm{TT}$ versus $\mathrm{KMC}$ alone in improving anthropometric and physiologic parameters and decreasing morbidities and hospital stay in low birth weight (LBW) infants.

Methods. Stable LBW infants ( $<2000 \mathrm{~g}$ ) admitted in the KMC ward were randomized to either intervention (KMC+TT) or control (KMC only) group. The KMC+TT group underwent thrice daily touch therapy sessions until discharge.

Results. A total of 50 infants were included in the study. Baseline characteristics between the two groups were comparable. There was a significant increase in weight gain (grams/kg/day) in the KMC+TT group compared to $\mathrm{KMC}$ only. Physiologic parameters like heart rate, respiratory rate, and temperature were significantly better in the $\mathrm{KMC}+\mathrm{TT}$ group. More infants in the KMC only group were transferred to NICU care, while more $\mathrm{KMC}+\mathrm{TT}$ infants went home against advice. Hospital stay was similar between the two groups.
\end{abstract}

Conclusion. Touch therapy added to $\mathrm{KMC}$ is more effective than $\mathrm{KMC}$ alone in improving weight gain and physiologic parameters. It is associated with lower morbidities, although it did not affect the hospital stay.

Key Words: Kangaroo Mother Care, touch therapy, low birth weight

Corresponding author: Rocamia F. Rasalan-Fermin, MD Mariano Marcos Memorial Hospital and Medical Center Barangay 6, San Julian, City of Batac, llocos Norte, Philippines

Email: miatus22@yahoo.com

\section{INTRODUCTION}

Approximately 15 percent of births globally resulted in babies with low birth weights (LBW), and $96.5 \%$ of these LBW births occurred in developing countries. ${ }^{1}$ Of these LBW infants, 30\% died either before stabilization or during the first twenty-four hours of life. ${ }^{1}$ The World Health Organization (WHO) defined low birth weight infants as having birth weights $<2,500$ grams and very low birth weight infants as having birth weights $<1,500$ grams. $^{2}$ In the Philippines, disorders related to short gestation and LBW, not elsewhere classified, ranked $7^{\text {th }}$ among the top ten leading causes of infant mortality in 2013. ${ }^{3}$ Although LBW was rarely a direct cause of death, it indirectly contributed to $60-80 \%$ of neonatal deaths. The leading causes of neonatal death were birth asphyxia, infection, preterm birth, or babies born small for gestational age (SGA).,5 
In the last ten years (2005-2015), LBW infants made up $20 \%$ of all deliveries in our institution.

Optimal care for preterm or LBW infants may be achieved by the balance of simple interventions like Kangaroo Mother Care (KMC) and touch therapy (TT) and sophisticated technological advancements such as incubators and radiant warmers, with the common goal of ensuring safety, comfort, and improved survival of the newborn infant. ${ }^{5,6}$

KMC is an innovative method developed in 1978 as an alternative to traditional care methods for the LBW baby, addressing the problems of overcrowding, insufficient and expensive resources, and increased morbidity and mortality in neonatal intensive care units. ${ }^{6}$ In 1999, Dr. Socorro dL. Mendoza introduced KMC in our institution after training in Bogota, Colombia, supported by the Fundacion Canguro of Colombia. KMC consists of early and continuous skinto-skin contact, exclusive breastfeeding, and early discharge with adequate follow-up. ${ }^{5,6} \mathrm{It}$ is a safe, low-cost care method for LBW infants, particularly those weighing < 2000 grams at birth. A meta-analysis by Boundy showed that KMC compared to conventional care was associated with $36 \%$ lower mortality (RR 0.64, 95\% CI 0.46, 0.89). KMC decreased the risk of neonatal sepsis, hypothermia, hypoglycemia, hospital readmission, and increased exclusive breastfeeding. Newborns receiving KMC had lower mean respiratory rate and pain measures and higher oxygen saturation, temperature, and head circumference growth. ${ }^{7}$

Neonatal touch therapy was first introduced via ayurvedic medicine and flourished for decades in the Indian subcontinent. Touch therapy is referred to as "a metho-dological touch intended to stimulate the baby." Studies on touch therapy for preterm infants showed this technique to improve gains in weight, head circumference, and length, as well as improved sleep/wake states, decreased stress, improved skin integrity, increased development of the sympathetic nervous system, enhanced parent-infant bonding, and early discharge from the NICU. ${ }^{8,9}$

A Cochrane review by Vickers showed that infants randomized to massage (rubbing, stroking, or kinesthetic movements) significantly gained more weight than controls (weighted mean difference $5.1 \mathrm{~g}, 95 \%$ CI 3.5, 6.7). Information from 206 patients showed that the length of stay was decreased by 4.6 days in the massage group (95\% CI 2.6, 6.6). However, their performance on the Brazelton scales for habituation or motor maturity was not significantly different between the massage and control groups. ${ }^{10}$

Although there were several researches on the beneficial effects of KMC and TT, studied individually, there was no readily available data on the benefit of the two interventions combined. There was one study by Rangey and Sheth, which compared $\mathrm{KMC}$ versus TT. The results showed no difference in weight gain and hospital stay between the two. ${ }^{10}$

The purpose of this study was to determine the effectiveness of TT as an adjunct intervention to $\mathrm{KMC}$ with regards to weight gain and physiologic responses. The mothers in our KMC ward (40 beds) had been encouraged to perform massage on their infants. Still, the effect of the combination of KMC and TT has not yet been systematically studied and documented.

\section{OBJECTIVE}

To compare the effects of Kangaroo Mother Care and Touch Therapy (KMC+TT) versus KMC only (KMC) on the growth, physiologic parameters, and outcomes of LBW infants weighing less than 2000 grams enrolled in the KMC Program of the Dr. Jose Fabella Memorial Hospital (DJFMH).

\section{Specific Objectives}

1.To compare the effects of $\mathrm{KMC}+\mathrm{TT}$ versus $\mathrm{KMC}$ only on the growth of LBW infants $<2000$ grams at birth, in terms of weight, length, and head circumference.

2.To compare the effects of $\mathrm{KMC}+\mathrm{TT}$ versus $\mathrm{KMC}$ only on the following physiological measurements:

a.Oxygen saturation

b.Cardiac rate

c. Respiratory rate

d.Temperature

3.To compare the duration of hospitalization and $\mathrm{KMC}$ ward discharge outcomes between $\mathrm{KMC}+\mathrm{TT}$ versus $\mathrm{KMC}$ only groups.

\section{METHODS}

\section{Setting and Subjects}

This prospective randomized controlled trial was conducted in a KMC ward (Level II NICU) at the Dr. Jose Fabella Memorial Hospital (DJFMH) from April 8, 2016 to May 10, 2016. Neonates weighing less than 2000 grams, any gestational age, stable, with or without co-morbidities were included in the study. The study included critically ill LBW newborns on oxygen support, newborns with chromosomal abnormalities and life-threatening congenital anomalies, and newborns whose mothers were medically and psychologically sick, rendering them unfit to perform KMC.

\section{Ethical Considerations}

Informed consent was obtained from the mother before enrolling the infant in the study. The study had no anticipated harm since anthropometric measurements and physiologic monitoring were routinely done on all neonates admitted to the unit. Ethics approval for the conduct of the study was obtained from the DJFMH Research Ethics Committee.

\section{Sample Size}

To get an $\alpha=0.05, \beta=0.20$, and assumed difference in weight of 5.1 grams based on the study conducted by Vickers A; at least 21 subjects per group was needed. ${ }^{11}$ 


\section{Randomization}

After informed consent was obtained, the infants were randomly allocated using the random allocation software (RALLOC) into the KMC only group and the KMC plus touch therapy (KMC+TT) group, with 25 infants in each group.

\section{Procedure}

\section{KMC only group}

Mothers in this group were given a detailed explanation about KMC adaptation, preferably in the presence of family members. KMC was initiated as soon as the baby was physiologically stable. The mothers provided continuous skin-to-skin contact and secured the infant in position using a tube blouse made of soft expandable cloth. Mothers were encouraged to keep the baby in continuous KMC for at least 20 hours per day. ${ }^{3}$ The mothers were given a $\mathrm{KMC}$ monitoring sheet to record the duration of kangaroo care provided. If the mother could not fill up the sheet, a family member (father or relatives of the patient) who was with the mother in the ward accomplished the form. Compliance with the continuous $\mathrm{KMC}$ protocol was monitored every 2 hours by the nurse on duty.

\section{$K M C+T T$ group}

Mothers who were assigned to the intervention group were taught how to perform touch therapy following Field's protocol (Figure 1A-C), in addition to performing $\mathrm{KMC}$. Before the start of the touch therapy, the mother

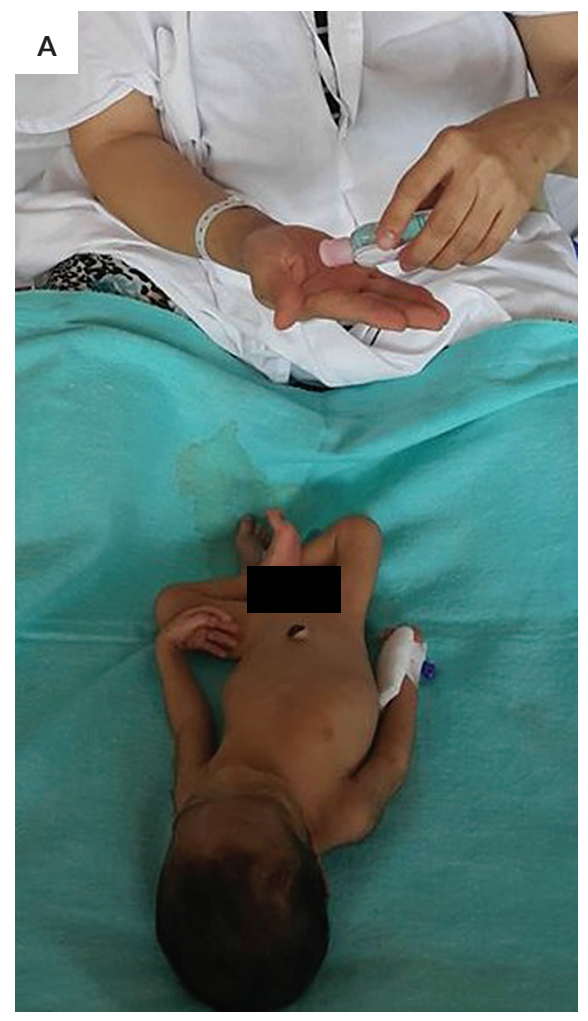

(A) Apply baby oil to the whole body of the infant prior to touch therapy.

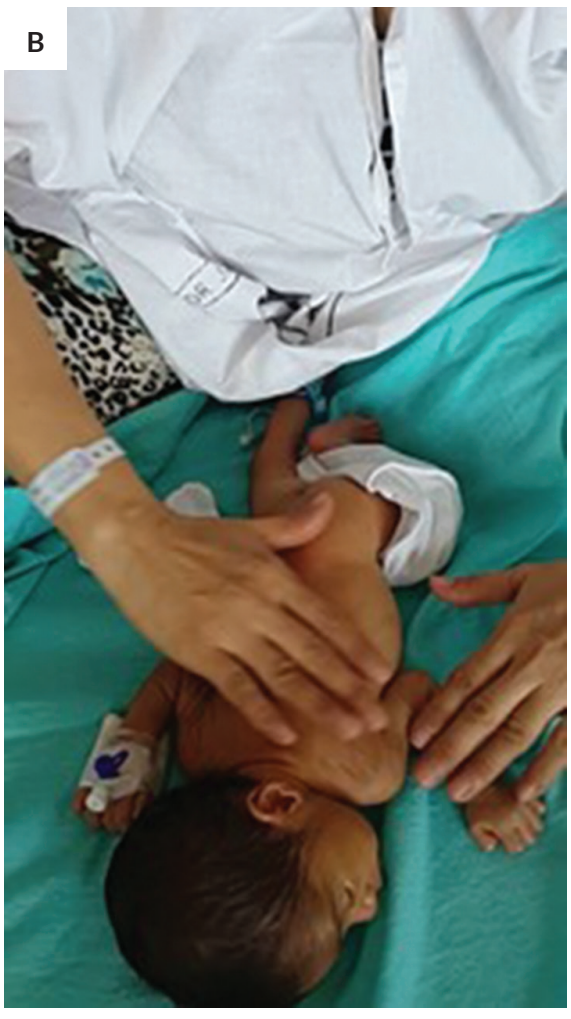

(B) Tactile:

Place infant in prone position. 5 strokes:

1. Head: downward strokes from crown to neck (alternating hands - $\mathrm{AH}$ )

2. Shoulders: horizontal outward strokes from middle of back towards shoulders (both hands - $\mathrm{BH}$ )

3. Back: downward strokes from neck to waist $(\mathrm{AH})$

4. Legs: downward strokes from thighs to ankles (BH)

5. Arms: horizontal outwards strokes from shoulder to wrist (BH)

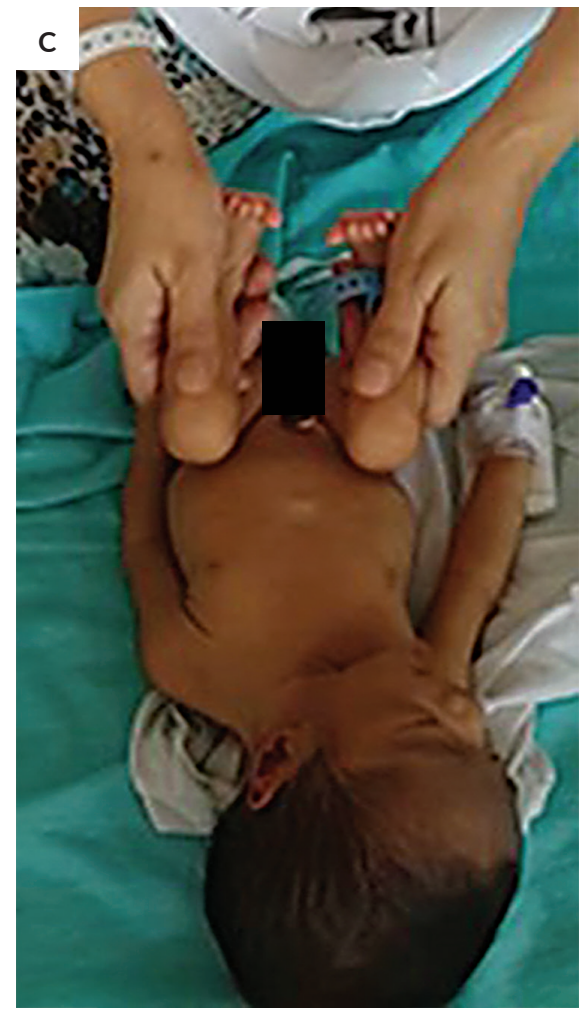

(C) Kinesthetic:

Place infants on their back. 6 movements for each:

1. Holding both forearms - flexed at elbow joints towards arms $(\mathrm{BH})$

2. Holding both legs - flexed at knee joint and towards abdomen.

Figure 1. Touch Therapy Procedure. (Photos were taken and posted with permission). 
applied baby oil to the body of her infant. The touch therapy session consisted of 2 parts. The initial part was for tactile stimulation, done while the infant was in the prone position. This consisted of providing five strokes each for the following body parts: (a) head - from crown to neck, (b) shoulders from the middle of back to arms, (c) back- from neck to waist, (d) legs-from top of thighs to ankles, and (e) arms from shoulder to wrist. The second part was the kinesthetic stimulation which was done with the infant in the supine position. This was done by repeatedly flexing each arm at the elbow joint, then flexing each leg at the knee joint. The touch therapy session was rendered for 15 minutes with 5 minutes of tactile stimulation followed by 5 minutes of kinesthetic stimulation and ending again with 5 minutes of tactile stimulation. ${ }^{12}$ Three touch therapy sessions were performed daily, provided either by a trained medical professional or the mother. Touch therapy sessions took place in a warm room with soft light and low noise levels. The massage was given between feeds and at least 45 minutes to 1 hour after a meal to avoid regurgitation or vomiting.

\section{Measurement Variables}

The primary outcome variables were growth parameters such as weight, length and head circumference. Secondary measures included physiologic parameters and neonatal morbidity defined as any untoward event occurring in the neonate: apnea, poor activity, poor suck, hypoglycemia, vomiting, and hyperthermia. The duration of hospitalization and the neonate's disposition (transferred back to the NICU III, discharged improved or brought home against medical advice) were also documented. All outcome measurements were obtained by a research assistant who was not part of the ward staff caring for the infants.

\section{Anthropometry}

1. Babies were weighed naked on an electronic weighing scale (Seca) immediately after birth. The weighing was subsequently done once daily at midnight until discharge. The weight was measured in grams, and the weighing machine was calibrated daily with 5-gram standard weight.

2. Length (L) measurement was taken with the infant in the recumbent position on the infantometer (Seca). The supine crown-heel length to the nearest centimeter was recorded with the help of an assistant.

3. Head circumference (HC) was measured by placing a flexible non-stretchable tape measure circumferentially from the infant's supraorbital ridge to the occiput (designated as the maximum occipitofrontal circumference).

\section{Physiologic Parameters}

1. Oxygen saturation was measured using the portable/ finger pulse oximeter, and saturation of $>95 \%$ is considered normal.
2. The cardiac rate was measured for 1 minute using a neonatal stethoscope, and a rate of $120-160$ beats/minute was considered normal.

3. Respiratory rate was measured by counting observed respirations for one full minute, and a rate of 30-50 cycles/minute was considered normal.

4. Temperature - was measured using a digital thermometer placed snugly in the axilla of the infant (normal value $36.5^{\circ} \mathrm{C}$ to $37.5^{\circ} \mathrm{C}$ ).

\section{Data Processing and Analysis}

Data was encoded and tallied in SPSS version 10 for Windows. Descriptive statistics were generated for all variables. For nominal data, frequencies and percentages were computed, and data were analyzed using Student's t-test. For numerical data, mean values $\pm \mathrm{SD}$ were calculated, and the Chi-square test was used for analysis.

\section{RESULTS}

A total of 50 subjects were included in the study. The participants were primarily preterm neonates with a mean gestational age of 34.0 weeks and a mean birth weight of 1773.5 grams. All the infants' weights were appropriate for age. The infants' mean age at enrolment was 7.5 days of life. Fifty-two percent of the infants had an initial diagnosis of neonatal pneumonia.

Baseline characteristics of the infants were comparable. There were no significant differences in sex, maturity, feeding, weight, head circumference, and length at birth upon $\mathrm{KMC}$ enrolment ( $p>0.05)$. There were no significant differences in maternal characteristics such as age, civil status, educational attainment, parity, age of gestation, and mode of delivery $(\mathrm{p}>0.05)$ (Table 1$)$.

Upon enrolment, there were no significant differences in the infants' weight (1776.40 vs. $1.684 .52 \mathrm{~g}, \mathrm{p}=0.09)$, head circumference ( 30.14 vs. $30.64 \mathrm{~cm} ; \mathrm{p}=0.88$ ), and length ( 42.7 vs. $43.18 \mathrm{~cm} ; \mathrm{p}=0.78$ ) between the KMC only and $\mathrm{KMC}+\mathrm{TT}$ groups. On the day of discharge, there were likewise no significant differences in the weight (1871 vs. $1848.04 \mathrm{~g} ; \mathrm{p}=0.66$ ), head circumference (30.06 vs. $30.98 \mathrm{~cm}$; $\mathrm{p}=0.56)$, and length ( 43.18 vs. $42.50 \mathrm{~cm} ; \mathrm{p}=0.40)$ between the $\mathrm{KMC}$ only and $\mathrm{KMC}+\mathrm{TT}$ groups (Table 2).

Comparing the mean increase in weights from enrolment to discharge, there was a significantly higher rise in weight in the $\mathrm{KMC}+\mathrm{TT}$ group (161.52 vs. $95.20 \mathrm{~g}$, $\mathrm{p}=0.02)$ compared with the KMC only group. Likewise, the mean increase in weight/day (29.94 vs. $16.18 \mathrm{~g} /$ day; $\mathrm{p}=0.04$ ) and mean a daily increase in weight $/ \mathrm{kg} /$ day $(16.90 \pm 15.60$ vs. $8.96 \pm 11.12 \mathrm{~g} / \mathrm{kg} /$ day; $\mathrm{p}=0.03$ ) were almost double in the $\mathrm{KMC}+\mathrm{TT}$ group versus the KMC only group (Table 3 ).

There was no significant difference seen in the oxygen saturations between the two groups on the physiologic parameters. However, significantly lower mean cardiac rates on enrolment (138.92 vs.149.40 bpm, $\mathrm{p}<0.001)$ and upon 
Effect of KMC with touch therapy

Table 1. Comparison of Infant and Maternal Characteristics between the KMC and the KMC+TT Group

\begin{tabular}{|c|c|c|c|}
\hline Characteristics & KMC Group $(n=25)$ & KMC + TT Group (n = 25) & p-value \\
\hline \multicolumn{4}{|l|}{ Infant Characteristics } \\
\hline \multicolumn{4}{|l|}{ Sex } \\
\hline Male & $14(56 \%)$ & $15(60 \%)$ & 0.77 \\
\hline Female & $11(44 \%)$ & $10(40 \%)$ & \\
\hline Birthweight (grams)* & $1810.60 \pm 182.50$ & $1797.17 \pm 259.14$ & 0.84 \\
\hline Pediatric aging (weeks)* & $34.44 \pm 1.85$ & $34.24 \pm 2.07$ & 0.72 \\
\hline Head circumference at birth $(\mathrm{cm})^{*}$ & $29.98 \pm 0.90$ & $30.30 \pm 1.45$ & 0.35 \\
\hline Length at birth $(\mathrm{cm})^{*}$ & $41.94 \pm 1.82$ & $42.30 \pm 2.11$ & 0.52 \\
\hline \multicolumn{4}{|l|}{ Initial diagnosis on enrollment } \\
\hline Pneumonia & 13 & 13 & 1.00 \\
\hline Sepsis & 9 & 10 & 0.77 \\
\hline RDS & 1 & 1 & 1.00 \\
\hline LBW/SGA & 1 & 1 & 1.00 \\
\hline Polycythemia & 1 & 0 & 1.00 \\
\hline \multicolumn{4}{|l|}{ Maturity and weight for age } \\
\hline Preterm AGA & $17(68 \%)$ & $19(76 \%)$ & \\
\hline Preterm SGA & $6(24 \%)$ & 2 (8\%) & 0.24 \\
\hline Term SGA & $2(3 \%)$ & $4(16 \%)$ & \\
\hline \multicolumn{4}{|l|}{ Manner of feeding } \\
\hline Breastfeeding (BF) & $13(52 \%)$ & $14(56 \%)$ & 0.77 \\
\hline Dropper Feeding (DF)+ BF & 2 ( $8 \%)$ & 0 & 0.48 \\
\hline DF+ Gavage Feeding (GF) & $1(4 \%)$ & $11(44 \%)$ & 1.00 \\
\hline GF & $7(28 \%)$ & 0 & 0.24 \\
\hline $\mathrm{GF}+\mathrm{BF}$ & $2(8 \%)$ & 0 & 0.48 \\
\hline \multicolumn{4}{|l|}{ Maternal Characteristics } \\
\hline Mothers' age (years)* & $24.20 \pm 5.33$ & $26.24 \pm 6.75$ & 0.24 \\
\hline \multicolumn{4}{|l|}{ Mother's marital status } \\
\hline Married & $4(16 \%)$ & $5(20 \%)$ & \\
\hline Single & $21(84 \%)$ & $20(80 \%)$ & 1.00 \\
\hline \multicolumn{4}{|l|}{ Mother's educational attainment } \\
\hline Elementary & $1(4 \%)$ & $2(8 \%)$ & \\
\hline High school undergraduate & $2(8 \%)$ & $2(8 \%)$ & \\
\hline High school graduate & $15(60 \%)$ & $17(68 \%)$ & \\
\hline College undergraduate & $2(8 \%)$ & 0 & 0.46 \\
\hline College graduate & $3(12 \%)$ & $4(16 \%)$ & \\
\hline Vocational & $2(8 \%)$ & 0 & \\
\hline \multicolumn{4}{|l|}{ Parity } \\
\hline G1P0 & $15(60 \%)$ & $10(40 \%)$ & \\
\hline G2P1 & $4(16 \%)$ & $7(28 \%)$ & \\
\hline G3P2 & $4(16 \%)$ & $6(24 \%)$ & 0.52 \\
\hline G4P3 & $1(4 \%)$ & 0 & \\
\hline G5P4 & $1(4 \%)$ & $1(4 \%)$ & \\
\hline G6P5 & 0 & $1(4 \%)$ & \\
\hline \multicolumn{4}{|l|}{ Manner of delivery } \\
\hline $\mathrm{CS}$ & $16(64 \%)$ & 13 (52\%) & \\
\hline NSD & $9(36 \%)$ & 12 (48\%) & 0.39 \\
\hline
\end{tabular}

${ }^{*}$ Mean $\pm S D$

Table 2. Comparison of Weight, Height and Length on Enrolment, and Discharge between the KMC only and the KMC+TT Group

\begin{tabular}{llccc} 
Anthropometric Measurements & Time Period & KMC Group $(\mathbf{n}=\mathbf{2 5})$ & KMC+TT Group $(\mathbf{n}=25)$ & p- value \\
\hline Age on Enrolment (Days of life) & & $7.9 \pm 1.25$ & $8.5 \pm 1.70$ & 0.10 \\
\hline Weight $(\mathrm{g})$ & Enrolment & $1776.40 \pm 144.34$ & $1684.52 \pm 203.45$ & 0.09 \\
& Discharge & $1871.60 \pm 151.33$ & $1848.04 \pm 214.96$ & 0.66 \\
\hline Head Circumference $(\mathrm{cm})$ & Enrolment & $30.14 \pm 0.93$ & $30.64 \pm 1.10$ & 0.88 \\
& Discharge & $30.06 \pm 1.43$ & $30.98 \pm 2.90$ & 0.56 \\
\hline Length $(\mathrm{cm})$ & Enrolment & $42.70 \pm 1.18$ & $43.18 \pm 1.46$ & 0.78 \\
& Discharge & $43.18 \pm 1.46$ & $42.50 \pm 2.85$ & 0.40 \\
\hline
\end{tabular}


Table 3. Comparison of Weight Gain between the KMIC and the KMC+TT Group

\begin{tabular}{lccc}
\multicolumn{1}{c}{ Weight Gain } & \multicolumn{1}{c}{ KMC Group $(\mathrm{n}=\mathbf{2 5})$} & \multicolumn{1}{c}{ KMC+TT Group $(\mathrm{n}=\mathbf{2 5})$} & $\mathrm{p}$-value \\
\cline { 2 - 4 } Mean Increase in weight from enrolment to discharge & $95.20 \pm 95.35$ & $161.52 \pm 99.59$ & 0.02 \\
\hline Mean weight gain/day & $16.18 \pm 20.56$ & $29.94 . \pm 26.01$ & 0.04 \\
\hline Mean weight gain/kg/day & $8.96 \pm 11.12$ & $16.90 \pm 15.60$ & 0.03 \\
\hline
\end{tabular}

Table 4. Comparison of Physiologic Status on Enrolment and Discharge between KMC only and KMC+TT Group

\begin{tabular}{llccc}
\hline Anthropometric Measurements & Time Period & KMC Group $(\mathbf{n}=\mathbf{2 5})$ & KMC+TT Group $(\mathbf{n}=\mathbf{2 5})$ & $\mathbf{p}$ - value \\
\hline Pulse oximeter $(\%)$ & Enrolment & $98.68 \pm 1.28$ & $98.24 \pm 1.16$ & 0.21 \\
& Discharge & $97.80 \pm 1.04$ & $97.90 \pm 0.74$ & 0.78 \\
\hline Cardiac rate $(\mathrm{bpm})$ & Enrolment & $149.40 \pm 7.48$ & $138.92 \pm 5.48$ & $<0.001$ \\
& Discharge & $144.00 \pm 5.74$ & $139.68 \pm 5.11$ & 0.006 \\
\hline Respiratory rate $(\mathrm{bpm})$ & Enrolment & $42.70 \pm 1.18$ & $43.18 \pm 1.46$ & $<0.001$ \\
& Discharge & $43.18 \pm 1.46$ & $42.50 \pm 2.85$ & 0.05 \\
\hline Temperature $\left({ }^{\circ} \mathrm{C}\right)$ & Enrolment & $42.70 \pm 1.18$ & $36.84 \pm 0.18$ & 0.02 \\
& Discharge & $43.18 \pm 1.46$ & $36.89 \pm 0.26$ & 0.05 \\
\hline
\end{tabular}

Table 5. Comparison of Weight Gain between the KMIC and the KMC+TT Group

\begin{tabular}{lccc} 
& KMC Group ( $\mathbf{n}=\mathbf{2 5})$ & KMC + TT Group (n = 25) & p-value \\
Infant Outcome & & & \\
$\quad$ Transferred back to NICU & $4(16 \%)$ & 0 & 0.01 \\
Discharged & $21(84 \%)$ & $20(80 \%)$ & \\
HAMA & 0 & $5(20 \%)$ & 0.80 \\
\hline $\begin{array}{l}\text { Duration of Hospitalization } \\
\text { Mean } \pm \text { SD (days) }\end{array}$ & $9.00 \pm 7.70$ & $8.52 \pm 6.20$ & 0 \\
\hline
\end{tabular}

discharge (139.68 vs. $144.00 \mathrm{bpm}, \mathrm{p}=0.006)$ were noted in the $\mathrm{KMC}+\mathrm{TT}$ compared with the $\mathrm{KMC}$ only group. The mean respiratory rate on enrollment was significantly higher in the $\mathrm{KMC}+\mathrm{TT}$ group (43.18 vs. $42.70 \mathrm{cpm}$, $\mathrm{p}<0.001)$ but was considerably lower upon discharge $(42.50$ vs. $43.18 \mathrm{cpm}, \mathrm{p}=0.05)$. Nonetheless, these values were not clinically significant. Temperature measurements were both significantly higher on enrolment $\left(36.84\right.$ vs. $36.71^{\circ} \mathrm{C}$, $\mathrm{p}=0.02)$ and upon discharge ( 36.89 vs. $\left.36.74^{\circ} \mathrm{C}, \mathrm{p}=0.05\right)$ in the $\mathrm{KMC}+\mathrm{TT}$ group compared with the KMC only group (Table 4).

Four patients in the KMC only group deteriorated and were transferred back to the NICU. The reasons for transfer were vomiting (2), fever (1), and apnea (1). On the other hand, none of the infants in the KMC+TT group developed further morbidities. However, five infants in the $\mathrm{KMC}+\mathrm{TT}$ group went home against medical advice (HAMA) due to family issues $(\mathrm{p}=0.01)$. The length of hospital stay did not significantly differ between the two groups (KMC+TT: 8.52 vs. KMC only: 9.0 days, $\mathrm{p}=0.80$ ) (Table 5).

\section{DISCUSSION}

One goal of neonatal management of the LBW infant is to realize postnatal growth equivalent to intra-uterine growth accumulation rates; non-nutritive strategies should be pursued and provide sustenance. ${ }^{13}$ There have been nume-rous studies on the improvement of growth using touch therapy or $\mathrm{KMC}$, but only a few studies have assessed the effectiveness of the two interventions together. Our study demonstrated that the daily weight gain of infants provided both KMC and TT was faster compared with KMC alone. Although not seen in our study due to the short hospital stay (8-9 days), other studies on KMC or TT, albeit not both, showed improvement in gains in length and head circumference. ${ }^{14-16}$ In a study by Scafidi, forty preterm infants who were provided with tactile/kinesthetic stimulation for 45 minutes daily for ten days showed a significantly faster weight gain than controls (34 vs. 28 g; $\mathrm{p}=0.03) .{ }^{17}$ Likewise, the study of Mathai also showed a higher weight gain among infants who underwent massage therapy ( 21.9 vs. $4.24 \mathrm{~g} / \mathrm{day}, \mathrm{p}=0.001) .{ }^{18}$ However, these two studies were on touch therapy alone and not the combination of KMC and TT such as in our study.

A study by Banker done in Western India compared the provision of KMC plus massage therapy versus conventional care (incubator or open care system) among LBW infants who were $<32$ weeks gestation. Similar to our study, the $\mathrm{KMC}+\mathrm{TT}$ group demonstrated better weight on discharge, fewer hypothermic episodes, and shorter hospital stays than the conventional care group. ${ }^{19}$ In Banker's study, the control group received conventional care, while in our 
research, our control received KMC. In our study, although the $\mathrm{KMC}+\mathrm{TT}$ group had a significantly higher temperature compared with the KMC only group, the difference was not clinically significant. Furthermore, there was no hypothermic episode observed in both the $\mathrm{KMC}+\mathrm{TT}$ and $\mathrm{KMC}$ only groups. $\mathrm{KMC}$ had long been established to facilitate thermoregulation through heat conduction from the mother's skin to the infants. The use of oil in touch therapy also improved thermoregulation by decreasing convection losses through the infant's skin.

Our study showed a significantly lower cardiac rate among the $\mathrm{KMC}+\mathrm{TT}$ group compared with the $\mathrm{KMC}$ only group. Providing touch therapy to a neonate stimulates his central nervous system that sets off a chain reaction; such as secretion of more serotonin (hormone affecting digestion, appetite, and sleep) and less cortisol and norepinephrine (secreted in response to stress), which would result in a lower cardiac rate and respiratory rate. ${ }^{20}$ In our study, the statistical differences in the respiratory rates between the two groups were not clinically relevant since these were within the normal range (42-43 breaths per minute). The oxygen saturations were also similar between the two groups. Although $52 \%$ of the recruited infants had an initial diagnosis of pneumonia and may have been tachypneic and oxygenrequiring, they were transferred to the $\mathrm{KMC}$ ward once they were stable and recovering.

In the study of Mendes and Procianoy in 2008, massage therapy in very low birth weight infants decreased the length of hospital stay (42 vs. 46 days, CI: 42, 56) and lower incidence of late-onset neonatal sepsis (10.8 vs. $38.3 \%$, $\mathrm{p}=0.005)$ compared with the control group (no massage). ${ }^{21}$ The reduction in hospital stay among the massage group might be attributed to the faster weight gain, improved sleepwake states, and reduced stress behaviors and activity after the massage therapy. In our study, there was no significant difference between $\mathrm{KMC}+\mathrm{TT}$ and $\mathrm{KMC}$ groups since both interventions, especially $\mathrm{KMC}$, have been shown to shorten hospital stay. ${ }^{6}$ Also, the KMC+TT group could have a longer mean duration of hospitalization if no infants were prematurely brought home against medical advice.

Four infants in the KMC group were transferred back for neonatal intensive care. Two had vomiting, one had apnea, and the last had a fever. The four were subsequently treated for sepsis. Although the majority of the infants; $88 \%(22 / 25)$ and $92 \%(23 / 25)$ of infants in the KMC only and $\mathrm{KMC}+\mathrm{TT}$ groups, respectively, were already diagnosed with pneumonia or sepsis from the start, there could be a protective role of touch therapy in addition to $\mathrm{KMC}$, against bacterial infection as seen in our study. In the meta-analysis of Conde-Aguedo, KMC significantly decreased the incidence of sepsis. ${ }^{6}$ Early and continuous skin-to-skin contact would colonize the infants' skin with maternal skin flora rather than hospital pathogens. The lower incidence of late-onset sepsis among infants who received touch therapy versus those who did not was an unexpected finding, as quoted in the study of Mendes and Procianoy. ${ }^{21}$ They hypothesized a possible improvement in the immune system, although its mechanism was not explained among those who received touch therapy in their study. KMC and TT may likely decrease sepsis incidence in different ways.

\section{CONCLUSION}

The addition of touch therapy to Kangaroo Mother Care may potentiate the known beneficial effects of KMC intervention. The combined intervention led to a faster weight gain, lower cardiac rate, better thermoregulation, and potentially fewer morbidities. Length of stay was the same whether infants were provided TT added to KMC or KMC alone. Touch therapy should be recommended as an adjunct intervention to Kangaroo Mother Care to LBW infants.

\section{Statement of Authorship}

All authors participated in the data collection and analysis and approved the final version submitted.

\section{Author Disclosure}

All authors declared no conflicts of interest.

\section{Funding Source}

The study has no funding support.

\section{REFERENCES}

1. WHO, Maternal, Newborn, Child and Adolescent Health Care of Preterm and/or Low-birth-Weight Newborn [Internet]. 2013 [cited 2015 Oct]. Available from:http://www.who.int/maternal_child_ adolescent/topics/newborn/care_of_preterm/en/

2. Infant Mortality: Ten (10) Leading Causes, Philippines, [Internet]. 2013 [cited 2015 Oct]. Available from: https://www.doh.gov.ph/ mortality

3. World health report 2005: Make Every Mother and Child Count. Geneva: WHO [Internet]. 2005 [cited 2015 Oct]. Available from: https://www.who.int/whr/2005/whr2005_en.pdf

4. Lawn JE, Mwansa-Kambafwile J, Horta BL, Barros FC, Cousens S. 'Kangaroo Mother Care' to Prevent Neonatal Deaths Due to Preterm Birth Complications. Int J Epidemiol. 2010 Apr; 39 (Suppl 1):i144-54.

5. Charpak N, Ruiz-Peláez JG, Figueroa de Calume Z. Current Knowledge of Kangaroo Mother Intervention. Curr Opin Pediatr. 1996 Apr;8(2):108-12.

6. Conde-Agudelo A, Díaz-Rossello JL. Kangaroo Mother Care to Reduce Morbidity and Mortality in Low Birth Weight Infants. Cochrane Database of Syst Rev. 2003;(2):CD002771.

7. Boundy EO, Dastjerdi R, Spiegelman D, Fawzi WW, Missmer SA, Lieberman E, et al. Kangaroo Mother Care, and Neonatal Outcomes: A Meta-analysis. Pediatrics. 2016 Jan;137(1).

8. Leonard J. Exploring Neonatal Touch Mind Matters: The Wesleyan Journal of Psychology. 2008; 3:39-47.

9. Mathai S, Fernandez A, Mondkar J, Kanbur W. Effects of Tactilekinesthetic Stimulation in Preterms: a Controlled Trial. Indian Pediatr. 2001 Oct;38(10):1091-8.

10. Rangey PS, Sheth M. Comparative Effect of Massage Therapy versus Kangaroo Mother Care on Body Weight and Length of Hospital Stay in Low Birth Weight Preterm Infants. Int J Pediatr. 2014;2014:434060. 
11. Vickers A, Ohlsson A, Lacy JB, Horsley A. Massage for Promoting Growth and Development of Preterm and/or Low Birth Weight Infants. Cochrane Database Syst Rev. 2004; 2004(2): CD000390.

12. Field TM, Schanberg SM, Scafidi F, Bauer CR, Vega-Lahr N, Garcia R, et al., Tactile/kinesthetic Stimulation Effects on Preterm Neonates. Pediatrics. 1986 May;77(5):654-8.

13. Ramanathan K, Paul VK, Deorari AK. Kangaroo Mother Care in Very Low Birth Weight Infants. Indian J Pediatr. 2001; 68:1019-23.

14. Cattaneo A, Davanzo R, Worku B, Surjono A, Echeverria M, Bedri A, et al. Kangaroo Mother Care for Low Birth Weight Infants: A Randomized Controlled Trial in Different Settings. Acta Paediatr. 1998; 87(9):976-985.

15. Hann M, Malan A, Kronson M, Bergman N, Huskisson J. Kangaroo Mother Care. S Afr Med J1999; 89: 37-39.

16. Kangaroo Mother Care. A Practical Guide. Department of Reproductive Health and Research. Geneva: World Health Organisation [Internet]. 2003 [cited 2015 Oct]. Available from: https://www.who.int/reproductivehealth/publications/maternal_ perinatal_health/9241590351/en/
17. Scafidi FA, Field TM, Schanberg SM, Bauer CR, Tucci K, Roberts J, et al. Massage Stimulates Growth in Preterm Infants: A Replication. Infant Behav Dev. 1990; 13(2):167-88.

18. Mathai S, Fernandaz A, Mondkar J, Kanbur W. Effects of Tactilekinesthetic Stimulation in Preterms: A Controlled Trial. Indian Pediatr 2001; 38:1091-8.

19. Banker, Deepa A. Kangaroo Mother Care (KMC) and Massage Therapy (MT): Prospective Randomized Controlled Trial of 103 Cases in Western India, OP31, 2015, p.69.

20. Field T, Hernandez-Reif M, Diego M, Schanberg S, Kuhn C. Cortisol Decreases and Serotonin and Dopamine Increase Following Massage Therapy. Int J Neurosci. 2005 Oct;115(10):1397-413.

21. Mendes EW, Procianoy RS. Massage Therapy Reduces Hospital Stay and Occurrence of Late-onset Sepsis in Very Preterm Neonates. J Perinatol. 2008 Dec;28(12):815-20. 